\section{Conditioned Discrimination of Electric Waves Differing Only in Form and Harmonic Content in the Electric Fish, Eigenmannia}

\author{
B. Kramer and G.K.H. Zupanc \\ Zoologisches Institut der Universität, D-8400 Regensburg
}

The individual frequencies of the continuous, wave-like electric organ discharges (EODs) of the South American knife fish Eigenmannia (Gymnotiformes, Teleostei) are remarkably constant at stable temperatures $[1,2]$ within a species-specific range of 240-600 Hz [3]. Playback experiments using tape-recorded EODs, presentations of sinusoidal electric stimuli and field observations seemed to indicate that the EOD frequency is a cue in species recognition [3, 4]. However, a later study of sympatric fish communities revealed, a signal's frequency is of limited use in species recognition due to extensive overlapping of species-specific ranges in EOD frequencies [5].

We tested whether Eigenmannia is sensitive to the finer detail of its EOD, especially the sexual dimorphism in waveform and harmonic content $[6,7]$. The male EOD resembles short pulses superimposed on a head-negative D.C. baseline containing higher harmonics or overtones which are strong compared to the fundamental (or EOD) frequency, while the female EOD almost resembles a sine wave containing weak, higher harmonics (Fig. $1 \mathrm{a}, \mathrm{b}$ ).

Four E. lineata $(11.6-13.2 \mathrm{~cm})$, obtained from a tropical fish dealer, were tested during the day. The test aquarium $(75 \times 40 \times 42 \mathrm{~cm}$ high; water conductivity $100 \pm 5 \mu \mathrm{S} \mathrm{cm}^{-1}$; temperature $27 \pm 1.5^{\circ} \mathrm{C}$ ) was provided with a centred porous pot (length $33 \mathrm{~cm}$; inner diameter $6.5 \mathrm{~cm}$; wall $1.2 \mathrm{~cm}$ ) the long axis of which was parallel to the smaller sides of the aquarium. An isolated fish remained in the porous pot most of the day ( $L: D, 12: 12)$. One electric dipole made of carbon rod electrodes (diameter $0.5 \mathrm{~cm}$; length $1.8 \mathrm{~cm}$; separation of the two vertically oriented carbon rods $7.8 \mathrm{~cm}$ ) was placed parallel and close to each end of the aquarium, that is, symmetrically to the left and right of a fish hiding in its shelter. Imitations of natural EODs were digitally synthesized by a specially designed microprocessor system [8] and presented through one of the dipoles. A function generator provided sine and sawtooth waves. Stimulus amplitudes (p-p) were kept constant among two signals within $5 \%$, in a range of $240-690 \mu \mathrm{V} \mathrm{cm} \mathrm{cm}^{-1}$ at the position of the fish's head in its shelter (measured with the fish removed; at least $50 \mathrm{~dB}$ above threshold of the conditioned response). Stimulus frequency was $50 \mathrm{~Hz}$ above the fish's EOD frequency in order not to evoke a jamming avoidance response (JAR, [1, 2]). Male EODs and sawtooth waves with their strong overtones contrasted with female EODs and especially sine waves with little or no harmonic content (Fig. 1, right).

The first step in a fish's training consisted of forming the association of "presence of an electric signal" with a food reward (one Chironomus mosquito larva) obtained at a feeder nearby the active dipole. Learning progress was monitored by measuring the time (s) from the onset of a sine wave signal to the fish's touching a feeder. The next step in the fish's training consisted of discriminating a rewarded signal (for example, sine waves) from an unrewarded one (for example, sawtooth waves). Mild punishment was necessary to establish discrimination because of the fishes' tendency to approach an active dipole independent of whether it presented a rewarded or an unrewarded signal. When appropriate, a fish was "punished" by approaching it gently with a plastic rod (without touching). A fish punished in this way returned to its shelter.

Discrimination conditioning was conducted in a randomized-blocks design [9]. A training block consisted of four training trials (signal no. 1, left; signal no. 1, right; signal no. 2, left; signal no. 2 , right; sequence determined by random permutation) with equal numbers of presentations of each out of two signals actually in use. A subse- quent test trial without food reward nor punishment completed one randomized block. The sequence of test trials was also determined by random permutation. The number of trials per day was between 30 (24 training and 6 test trials) and 40 (32 training and 8 test trials). The inter-trial interval was $5 \mathrm{~min}$. When the fish did not respond a signal was turned off after $150 \mathrm{~s}$. Stimulus amplitude was smoothly turned on or off by hand during 1/2 $\mathrm{s}$. Under these conditions discrimination was established during the first day of training in all fish.

The fish discriminated successfully between all pairs of signals tested: rewarded sine waves and unrewarded sawtooth waves $(P<0.01$ in each of four fish tested; Fig. 2a); rewarded sine waves and unrewarded male EODs $(P<0.001$ in each fish; Fig. 2b); rewarded female EODs and unrewarded male EODs $(P<0.01$ in each fish; Fig. 2c). A fourth experiment (not shown) consisted of reversing the roles of rewarded and unrewarded signals, as used in the second experiment, and immediately following it: now it was the dipole playing back rewarded male EODs which was approached faster than the one presenting unrewarded sine waves $(P<0.025$ in three out of four fish; $N \geq 12$ test trials per fish and waveform).

A simple cue enabling a fish to discriminate between two signals, as used in the present report, might be their differences in fundamental frequency intensities (compared to a sine wave of $0 \mathrm{~dB}$, fundamental frequency intensities were: $-4.1,-3.0$, and $0 \mathrm{~dB}$ for the sawtooth wave, the male and female EODs, respectively). A similar type of signal analysis was shown in Eigenmannia's JAR: the strength of JAR was correlated with the intensity of that stimulus harmonic which was close to the EOD fundamental frequency, and independent of stimulus waveform or harmonic content [7]. Therefore, in a fifth experiment the first experiment was repeated in a modified way, using an attenuated sine wave to match the weaker intensity of the fundamental frequency component of the sawtooth wave. All four fish tested still discriminated the rewarded sine wave from the unrewarded sawtooth wave of stronger peak-to-peak amplitude $(P<0.01$ in each fish; 

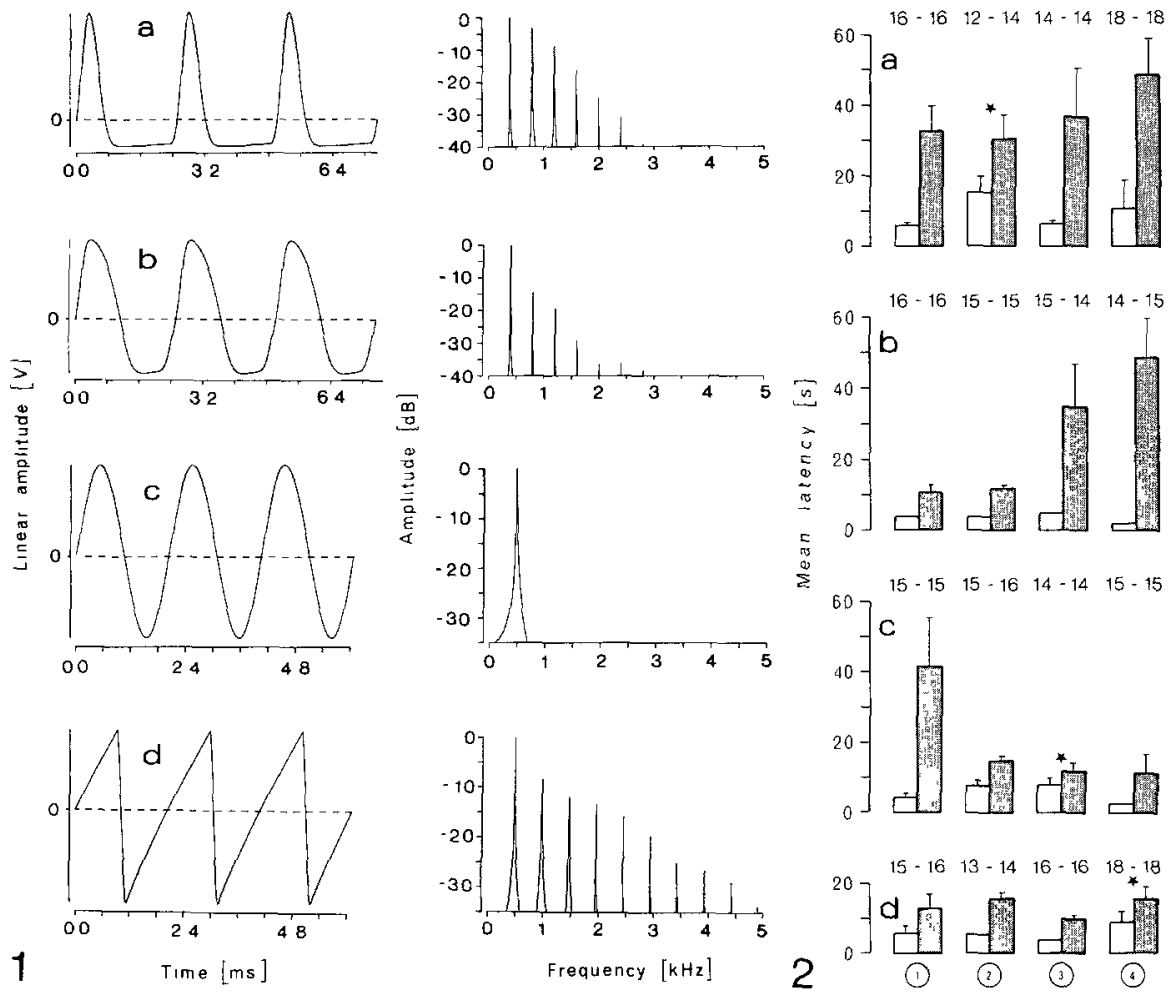

Fig. 1. Waveforms of electric signals (left) and Fourier amplitude spectra (right) as used in conditioned discrimination experiments with Eigenmannia lineata. The ordinates of the left diagrams are arbitrary linear amplitudes $(V)$, of the right diagrams amplitudes expressed as $\mathrm{dB}$ attenuation relative to the strongest spectral component of each waveform. a) Electric organ discharges (EODs) of a male Eigenmannia, as generated by a microprocessor-based system for the digital synthesis of EODs [8] (from [7]), b) digitally synthesized EODs of a female Eigenmannia (from [7]), c) sine waves and d) sawtooth waves generated by a function generator, all recorded from the water

Fig. 2. Mean latencies of four isolated Eigenmannia, measured from the onset of an electric signal presented through a dipole to a fish's touching a nearby feeder, in a food-rewarded conditioned discrimination experiment. Standard errors are either shown or are too small to be drawn. Individual fish numbers are on the bottom. The number of test trials is indicated above each column. Open columns rewarded signals; shaded unrewarded signals. The differences among all paired columns are significant at $P<0.001$ (except $*$ where $P<0.01$; one-tailed Mann-Whitney U-test). The rewarded and unrewarded signals, respectively, were in a) sine and sawtooth waves; b) sine waves and male EODs; c) female and male EODs; d) sine and sawtooth waves of matched intensities of their fundamental frequency components, hence different peak-to-peak amplitudes (unlike the other pairs of signals). Note that in each fish, its latency to approach a dipole presenting a rewarded signal was much shorter than when the dipole presented an unrewarded signal. Experiment c) shows discrimination of female from male EODs both having harmonic content. Experiment d) shows that the fish must have recognized categorical differences in the stimuli presented, not simply intensity differences in the fundamental frequency components

Fig. 2d). This shows that the fish indeed recognized categorical differences in the stimuli tested.

What is it that enables Eigenmannia to recognize categorical differences in electric signals? According to one hypothesis [10] the fish are sensitive for differences in the intervals between zero-crossings of the two half-waves of a signal cycle. These intervals are symmetric (equal) in a sine or sawtooth wave (Fig. 1c, d), but markedly asymmetric (unequal), for example, in the male EOD (Fig. 1a). Male EODs might therefore have been discriminated from sine waves by their pattern of zero-crossings intervals. However, that type of signal analysis can be ruled out in the first and the fifth experiment where the fish discriminated sine waves from sawtooth waves although they were identical regarding their intervals between zero-crossings. Variation of intervals between zero-crossings by varying the phase difference between harmonic signal components, that is, the form of the complex wave, did not affect the JAR [7].

Therefore, in the present study the fish probably noticed the differences of the signals in harmonic content. Experiments in progress test the hypothesis whether Eigenmannia discriminates electric signals differing in harmonic content in a way, for example, similar to timbre perception in the auditory system (for example, [11]). One of the sensory requirements of timbre perception, a set of differently tuned electroreceptors in the same fish, is met according to [12] (see also discussion in [7]).

Supported by the Deutsche Forschungsgemeinschaft (SFB4/H1), and a student fellowship from the FriedrichEbert-Foundation (Bonn) to G.Z. Prof. G. Neuweiler (University of Munich) and B.O. Bratton kindly criticized the manuscript.

Received April 17 and June 18, 1986

1. Watanabe, A., Takeda, K.: J. exp. Biol 40, 57 (1963)

2. Bullock, T.H., Hamstra, R.H., Scherch, H.: J. Comp. Physiol. 77, 1 (1972)

3. Hopkins, C.D.: Behaviour 50, 270 (1974)

4. Hopkins, C.D., Heiligenberg, W.: Behav. Ecol. Sociobiol. 3, 113 (1978)

5. Kramer, B., Kirschbaum, F., Markl, H., 1n: Sensory Physiology of Aquatic Lower Vertebrates, p. 195 (T. Szabo, G. Czeh, eds.). Budapest: Pergamon Press/ Akademiai Kiado 1981

6. Kramer, B.: Verh. Dtsch. Zool. Ges. 1983,170

7. Kramer, B.: J. exp. Biol. 119, 41 (1985)

8. Kramer, B., Weymann, D.: submitted

9. Cochran, W.G., Cox, G.M.: Experimental Designs. London-Sydney: Wiley 1968

10. Gottschalk, B., in: Sensory Physiology of Aquatic Lower Vertebrates, p. 255 (T. Szabo, G. Czeh, eds.). Budapest: Pergamon Press/Akademiai Kiado 1981

11. Roederer, J.G.: Introduction to the Physics and Psychophysics of Music. New York-Heidelberg-Berlin: Springer 1975

12. Viancour, T.A.: J. Comp. Physiol. 133, 317 (1979) 